\title{
Validation of the Malay 3-Minute Diagnostic Interview for Confusion Assessment Method in a surgical population
}

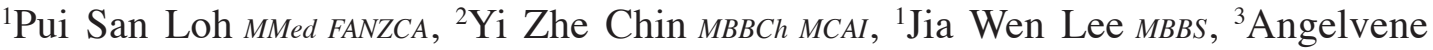 \\ Wong $M D,{ }^{1}$ Marzida Mansor MBbS MAnaes, ${ }^{4}$ Chong Guan Ng PhD MPsychMed, ${ }^{5}$ David Cowan \\ $M D F R C P(C),{ }^{6}$ Matthew TV Chan PhD FANZCA, ${ }^{1}$ Chew Yin Wang mBChB FRCA \\ ${ }^{1}$ Department of Anaesthesiology, Faculty of Medicine, University of Malaya, Kuala Lumpur; ${ }^{2}$ Department \\ of Anaesthesiology and Intensive Care Unit, Sarawak General Hospital, Jalan Hospital, Kuching, \\ Sarawak; ${ }^{3}$ Psychiatry Department, Hospital Sibu, Sibu, Sarawak; ${ }^{4}$ Department of Psychological \\ Medicine, Faculty of Medicine, University of Malaya, Kuala Lumpur, Malaysia; ${ }^{5}$ Department of \\ Medicine, McMaster University, Hamilton, Ontario, Canada $;{ }^{6}$ Department of Anaesthesia and Intensive \\ Care, The Chinese University of Hong Kong, Hong Kong Special Administrative Region, China
}

\begin{abstract}
Background: Delirium is a common postoperative complication among elderly which can be easily missed and leads to poorer outcomes. The 3-Minute Diagnostic Assessment for Confusion Assessment Method (3D-CAM) is a short and structured tool to assess delirium by healthcare staff with minimal training. This study aimed to validate the translated Malay 3D-CAM (M3D-CAM) in postoperative surgical patients. Methods: In this prospective diagnostic study, 3D-CAM was translated into Malay and two assessors ( 1 and 2) independently interviewed surgical patients above 65 years old with M3D-CAM on postoperative day one. A psychiatrist diagnosed postoperative delirium according to the Diagnostic and Statistical Manual of Mental Disorders 5th Edition (DSM-5) as the reference standard. The sequence of examinations was done randomly with all results blinded to each other and the diagnostic characteristics of M3D-CAM analysed with $\kappa$ coefficient used to evaluate reliability. Results: A total of 427 patients were screened, 111 recruited with a final 100 paired interviews completed. Their mean age was $72( \pm 6)$ years old. Two-thirds of patients were proficient in Malay and English, therefore assessed in both 3D-CAM and M3D-CAM. Delirium was identified in $11 \%$ and $12 \%$ of patients by assessors 1 and 2 respectively while compared to DSM-5, M3D-CAM had $80 \%$ and $90 \%$ sensitivity with $96.7 \%$ and $97.7 \%$ specificity. M3D-CAM had excellent inter-rater reliability (85\%), substantial parallel reliability (70\%) and features 1 and 3 with substantial parallel agreement $(\mathrm{p}<0.001)$.

Conclusion: This study demonstrated that M3D-CAM is reliable and valid for delirium assessment in the postoperative setting.
\end{abstract}

Keywords: Delirium, Confusion Assessment Method, 3-minute diagnostic, 3D-CAM, Malay 3D CAM, validation

\section{INTRODUCTION}

Delirium is a major contributor to postoperative morbidity and mortality in older adult patients after surgery. ${ }^{1,2}$ According to the Diagnostic and Statistical Manual of Mental Disorders 5th Edition (DSM-5), delirium is a neuropsychiatric disorder with acute disturbance in attention and cognition, that is unexplained by another preexisting neurocognitive condition. ${ }^{3}$ Clinically, postoperative delirium (POD) manifests as hypoactive, hyperactive or mixed psychomotor forms that commonly occur within the first three days after surgery. ${ }^{4-6}$ Despite awareness of its importance, the diagnosis is still commonly overlooked and under-diagnosed, with an overall estimated $12-35 \%$ detection rate especially in patients with underlying dementia or hypoactive behavioural changes. ${ }^{4,-9}$

Although the gold standard in diagnosing delirium had been those set by DSM-5, unfamiliarity and the lack of training frequently contributed to the under-detection of this

Address correspondence to: PS Loh, Department of Anaesthesiology, Faculty of Medicine, University of Malaya, 50603 Kuala Lumpur, Malaysia. Email: lohps@ummc.edu.my

Date of Submission: 12 April 2021; Date of Acceptance: 26 July 2021

https://doi.org/10.54029/2021xfd 
condition..$^{10}$ To overcome this difficulty, more than 20 published screening instruments had been developed to improve the practicality for detection of delirium with good diagnostic characteristics..$^{4,10,11}$ In 1990, Inouye et al. developed the Confusion Assessment Method (CAM) for a brief, accurate and standardized instrument for detecting delirium by medical staffs who did not undergo extensive training in psychiatry. It is a widely used screening tool with a high sensitivity of $94 \%$, specificity of $89 \%$ and excellent inter-rater reliability in many high-quality studies., ${ }^{411,12}$ The use of CAM had also been extended by translation into 12 different languages and adapted for use in the intensive care unit (ICU), emergency departments and nursing homes. ${ }^{4}$

However, CAM has its own set of challenges such as relying on substantial interviewer training as a pre-requisite, reliance upon the skill of the rater and a large variability for operationalizing each diagnostic feature. To overcome these issues, an accelerated assessment using the CAM algorithm was developed by Marcantonio et al. for brevity and ease of administration with a structured format. The abbreviated version, a new 3-Minute Diagnostic Assessment for CAM-defined delirium, otherwise known as 3D-CAM proved to be highly reproducible and valid for diagnosing delirium as it is specifically constructed for assessments by trained nonpsychiatric personnel in general medical patients, above 80 years old with multiple comorbidities and a high burden of baseline cognitive impairment. ${ }^{7}$ The use of 3D-CAM has the potential to improve the recognition of delirium in high risk patients, but to do so widely, it needs to be translated and validated in non-English languages just like CAM previously.

In this study, the primary aim was to translate and develop a version of 3D-CAM in Malay (M3D-CAM) with a secondary aim of validating this tool in our older adult surgical patients.

\section{METHODS}

This was a prospective cohort study to validate M3D-CAM in a multiracial surgical patient population of a tertiary referral university-based hospital. The study protocol was approved by Medical Research Ethics Committee and details were reported in ClinicalTrials.gov (NCT03366896). Written informed consent was obtained from all participants or their designated surrogates before surgery.

\section{Translation}

The first phase involved cultural adaptation of 3D-CAM into M3D-CAM according to existing guidelines on translation. ${ }^{13}$ Two translators, both anaesthetists proficient in English and Malay (YZ and JW) first performed the forward translation independently. Then, both discussed and merged the copies into a single draft and evaluated again together.

After ethics approval, the first M3D-CAM draft was tested among 20 bilingual healthcare providers from different fields and 5 bilingual patients above 65 years old in the pre-operative anaesthetic clinic. Subsequently, a detailed discussion was made amongst the panel of authors not involved in the first translation (authors CY and $\mathrm{CG}$ ) to appraise the ease of understanding all instructions, content, and wordings. Based on the feedbacks from the panel and the first batch of 25 participants, adjustments and refinements were made to form the second draft M3D-CAM.

Following this, the second draft M3D-CAM was back translated to English (PS and MM) individually to check for fidelity. The panel reconvened to compare the back translation with the original 3D-CAM and formed a finalized version of M3D-CAM that was approved by the original author. (Figure 1)

\section{Participants and enrolment}

Patients above 65 years old who were scheduled for elective and semi-urgent surgery within a period of 3 months in this institution were included in the study. We excluded patients who refused to participate, were unable to communicate in Malay, were planned for postoperative ventilation in ICU, had hearing or speech impairment and preoperative diagnosis of delirium, psychosis, or Glasgow Coma Scale (GCS) of $\leq 11 / 15$. After obtaining written informed consent, details of baseline characteristics and surgical data were collected.

\section{Validity and reliability testing}

The second phase in this study involved Assessor 1 (YZ) and Assessor 2 (a research assistant) for reliability testing. Both were trained in administering 3D-CAM by $\mathrm{CG}$, a psychiatrist before conducting delirium assessment. All assessments were performed in random order by Assessors 1,2 or psychiatrist (AW) within less than an hour apart. Each would approach patients independently in the surgical wards on postoperative day one and all three were blinded 


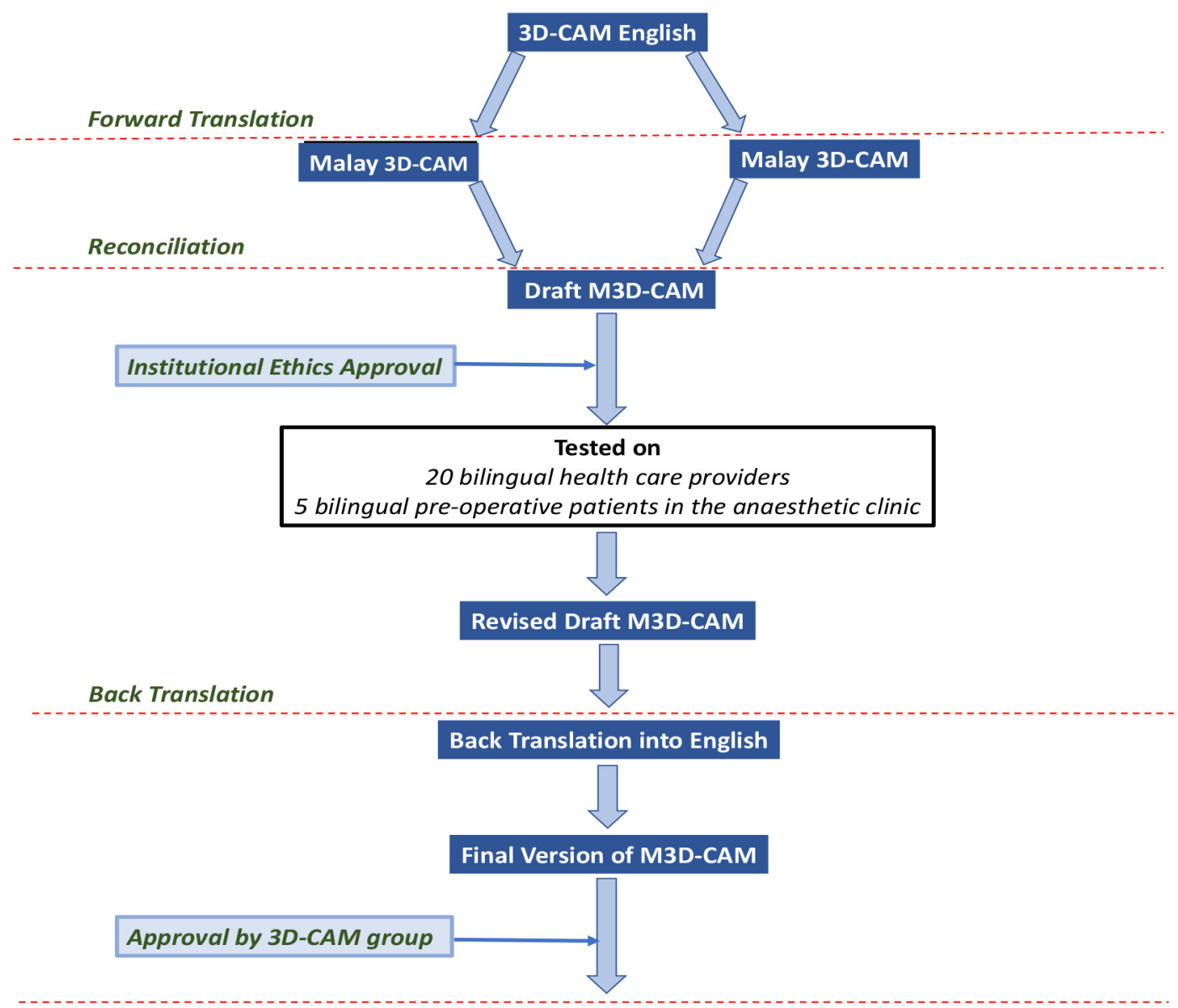

ACTUAL STUDY

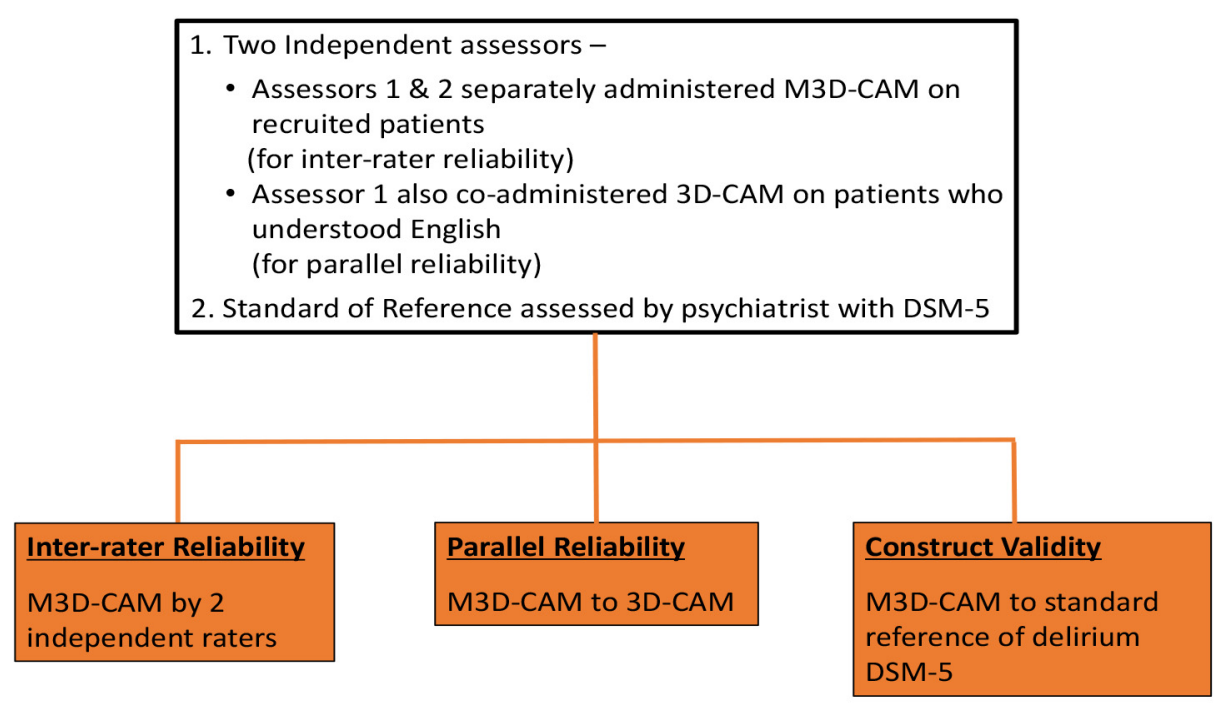

Figure 1. Methodology flowchart. 
to each other's results. Assessor 1 interviewed patients with M3D-CAM and the original 3D-CAM while Assessor 2 interviewed the same patient with M3D-CAM only. To conduct validity testing, AW evaluated the patients according to criteria for delirium in DSM-5. If patients were found to have delirium, the diagnosis was confirmed with a senior psychiatrist (CG) and the primary surgical team was then alerted.

\section{Sample size calculation and data analysis}

Sample size was calculated based on the approach of subject to item ratio of $5: 1{ }^{14}$ Therefore, with 20 items in M3D-CAM, the number of patients calculated would be 100. In addition, given the estimated incidence of delirium in our institution among non-cardiac surgical population $>65$ years old was $15 \% .{ }^{15}$ Assuming a width of $95 \%$ confidence interval, we needed to enrol 103 and 19 patients for sensitivity and specificity set at $80 \%$ respectively. The higher number of 103 was chosen and considering a 5\% drop-out rate, the calculated sample size was 108 with the figure rounded up to a total of 110 patients. ${ }^{16,17}$

\section{Data analysis}

All data analyses were performed using IBM software SPSS statistics version 22 package for Windows. Descriptive statistics was performed on baseline characteristics and presented as number and percentage for categorical data. The diagnostic characteristics of M3D-CAM were matched to a reference standard based on DSM-5 criteria for validation and explored as sensitivity, specificity, positive predictive (PPV) and negative predictive values (NPV). The use of Cohen's $\kappa$-coefficient evaluated both inter-rater reliability for M3DCAM results by Assessors 1 and 2 together with parallel reliability for the original 3D-CAM by Assessor 1 with M3D-CAM results by Assessor 2. Statistical significance was considered at a two-tailed $\mathrm{p}$ value of less than 0.05 .

\section{RESULTS}

\section{Patient characteristics}

From 26th June 2016 until 26th September 2016, a total of 437 patients were screened for eligibility from elective surgical lists. Electronic medical records were preliminarily evaluated for suitability to be included with a final number of 110 patients approached and recruited with informed consent. Ten patients dropped out after enrolment because they were discharged before completing reviews by all 3 researchers, refused to be interviewed postoperatively or had their surgery cancelled. A final number of 100 patients were studied within this period as shown in Figure 2.

Their mean age was $72( \pm 6)$ years old, with mostly females (58\%) and a multi-ethnic population of $46 \%$ Chinese, $34 \%$ Malay and $18 \%$

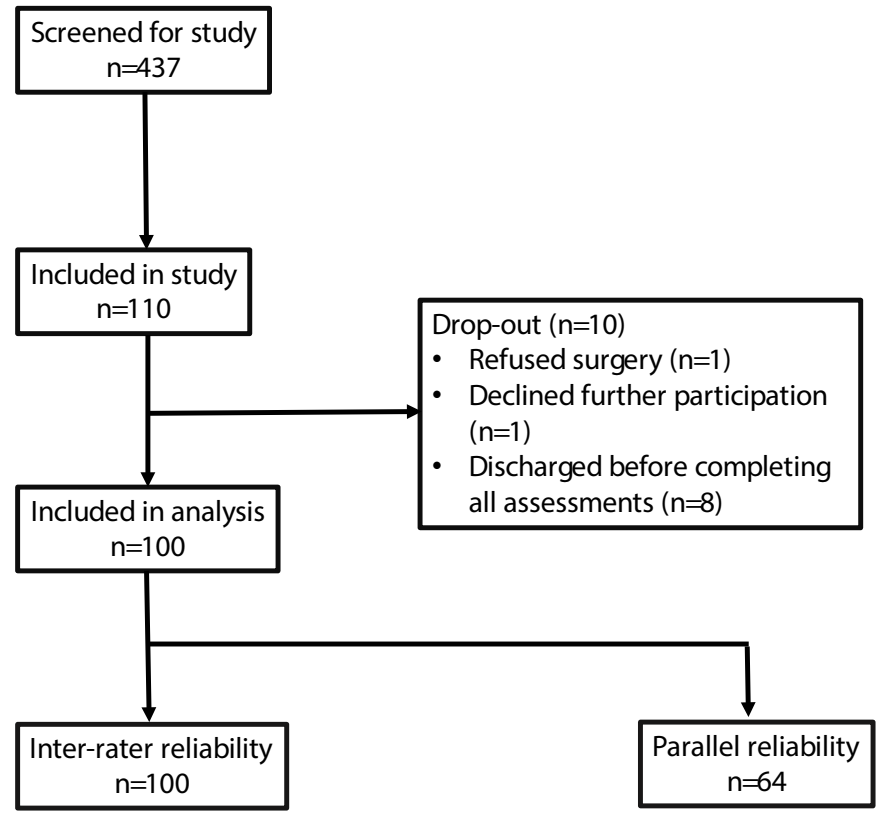

Figure 2. Consort diagram of study. 
Table 1: Demographic characteristics of the study population

\begin{tabular}{lc}
\hline Characteristics $(\mathbf{N}=\mathbf{1 0 0})$ & \\
\hline Age, mean (SD) & $71.78(5.51)$ \\
Female n (\%) & $58(58 \%)$ \\
Race, $\mathrm{n}(\%)$ & $34(34 \%)$ \\
Malay & $46(46 \%)$ \\
Chinese & $18(18 \%)$ \\
Indian & $2(2 \%)$ \\
Others & $64(64 \%)$ \\
Language - understood both Malay and English, n $(\%)$ & $1(1 \%)$ \\
Severe sensory Impairment - Vision, n (\%) & \\
Surgical discipline, $\mathrm{n}(\%)$ & $45(45 \%)$ \\
Orthopaedics & $20(20 \%)$ \\
General surgery & $8(8 \%)$ \\
Urology & $7(7 \%)$ \\
Gynaecology & \\
Others & \\
Urgency of operation, $\mathrm{n}(\%)$ & $65(65 \%)$ \\
Elective & $35(35 \%)$ \\
Semi-Emergency & \\
\hline
\end{tabular}

Indian (Table 1).Almost two-thirds were proficient in both Malay and English. Ten patients (10\%) were diagnosed to have delirium postoperatively by the psychiatrist with DSM-5; 8 of them $(80 \%)$ displayed hypoactive features while the remaining 2 showed hyperactive behaviours. Using the M3DCAM, Assessors 1 and 2 identified $11 \%$ and $12 \%$ respectively with delirium. Assessor 1 diagnosed $7.8 \%$ delirium amongst the 64 patients who could be tested with 3D-CAM in English. The overall duration of the interviews with M3D-CAM by both assessors were less than 5 minutes.

\section{Diagnostic test characteristics}

The validity of M3D-CAM was examined by comparing the detection of postoperative delirium of each assessor using M3D-CAM to the reference standard of diagnosis by a psychiatric expert. Assessor 1 achieved a sensitivity of $80 \%$ whilst Assessor 2 achieved 90\% (Table 2). Specificity of $96.7 \%$ and $97.7 \%$ were respectively found by the assessors administering M3D-CAM. In comparison, the sensitivity was $60 \%$ and specificity $96.6 \%$ in a subset of 64 participants who understood both languages, English and Malay when 3D-CAM was also used (Table 2).

Table 2: Diagnostic characteristics of M3D-CAM

\begin{tabular}{lcc}
\hline \multicolumn{4}{l}{ Diagnostic test characteristics of M3D-CAM compared to the reference standard (N=100) } \\
\hline & Assessor 1 & Assessor 2 \\
Sensitivity, \% (95\% CI) & $80(60-100)$ & $90(71-109)$ \\
Specificity, \% (95\% CI) & $96.7(93-100)$ & $97.7(94.6-100)$ \\
Positive predictive value, \% (95\% CI) & $72.7(46-99)$ & $81.8(59-104)$ \\
Negative predictive value, \% (95\% CI) & $97.7(94-100)$ & $98.9(96.7-101)$ \\
\hline Diagnostic test characteristics of M3D-CAM compared to the reference standard (N=64) \\
\hline Sensitivity, \% (95\% CI) & $60(20-102)$ \\
Specificity, \% (95\% CI) & $96.7(92-102)$ \\
Positive predictive value, \% (95\% CI) & $60(20-102)$ \\
Negative predictive value, \% (95\% CI) & $96.6(92-101)$ \\
\hline
\end{tabular}




\section{Reliability characteristics}

Overall, M3D-CAM had a near perfect interrater reliability of $85 \%$ in diagnosing delirium according to Landis and Koch's measurement of observer agreement. ${ }^{18}$ On evaluating reliability of individual M3D-CAM features to detect delirium, feature 2 scored moderate agreement ( $\kappa$-coefficient 0.53 ) whereas remaining features of 1, 3 and 4 scored substantial agreement (Table 3).

In addition, the parallel reliability between M3D-CAM compared to its original version was a substantial agreement of $70 \%$. Again, on evaluating reliability of individual 3D-CAM features to detect delirium, feature 2 showed moderate parallel agreement while features 1 and 3 have substantial parallel agreement of $65 \%$ and $71 \%$ respectively (Table 3 ). $\kappa$-values of feature 4 could not be determined because the number of positive responses was the same in 3D-CAM and Malay 3D-CAM.

\section{DISCUSSION}

The results of this study showed that M3D-CAM is a reliable screening instrument for POD in elderly patients. With M3D-CAM, the assessors identified an incidence of 11-12\% POD, a figure which corresponded well to the incidence of POD in a local study conducted with different assessment instruments (4-Abbreviation Test and CAM) in a similar cohort of non-cardiac, elderly surgical population. ${ }^{15} \mathrm{~A}$ similar incidence rate has also been reported in a study of a neighbouring country. ${ }^{19}$ However, the incidence of POD in this group of ageing population is slightly lower than the reported $13-50 \%$ in a recent systematic review on the incidence and outcome of delirium in non-cardiac surgery. ${ }^{4}$ Plausible explanations of a relatively lower POD incidence in our study were due to the exclusion of perioperative Intensive Care Unit (ICU) patients and those who underwent emergency operations. Both groups of patients have consistently shown to result in higher POD rates. ${ }^{4,20}$ In this study, the majority of affected patients presented clinically with hypoactive delirium with features dominated by symptoms of drowsiness and inactivity compared to hyperactive delirium, which would have been mainly symptoms of restlessness and agitation. ${ }^{21}$ Hypoactive presentation has been the main reason for the under detection of POD in the literature..$^{22,23}$

Older patients with delirium have an increased risk of admission to long term care or institutionalization upon discharge, lengthy and costly hospital stays with higher mortality rates while persistent delirium symptoms may lead to cognitive decline and dementia., $4,52,21,24-26$ Many studies have reported that early detection of postoperative delirium is vital as prompt measures can be taken to reduce its complications, including recognizing and treating underlying medical factors, optimizing the environment or analgesics and lastly, pharmacological treatment for recalcitrant postoperative delirium..$^{5,8,27,28}$ Hence, finding useful assessment instruments with validated translations in non-English languages will facilitate the diagnosis of delirium and widen the use in different populations. ${ }^{12}$

Just like the original 3D-CAM, the aim to be a short, structured diagnostic assessment with strong performance characteristics was achieved with M3D-CAM. Both sensitivity and specificity when validated against the reference standard were high (80-90\% and $>96 \%$ respectively) and these were similar to the findings of Marcantonio et al. and the Chinese version of 3D-CAM (3D-CAMCN). ${ }^{7,29}$ The slightly lower sensitivity compared to specificity was not unlike the findings for 3D-CAM-CN in surgical ICU patients. This was attributed to lower true positives in the postoperative setting because of the inherent challenge faced when recovering patients were interviewed after surgery. Distinguishing inattention or fluctuating changes of consciousness was difficult under the influence of uncontrolled pain or sedative effects of analgesic drugs. The

Table 3: Reliability agreement of both M3D-CAM and 3D-CAM in our study population

\begin{tabular}{|c|c|c|c|c|}
\hline \multirow[b]{2}{*}{ Features } & \multicolumn{2}{|c|}{ M3D-CAM (N=100) } & \multicolumn{2}{|c|}{ 3D-CAM $(\mathrm{N}=64)$} \\
\hline & К-value & $p$-value & א-value & $p$-value \\
\hline 1 - Acute onset and fluctuating course & 0.687 & $<0.001$ & 0.646 & $<0.001$ \\
\hline 2 - Inattention & 0.529 & $<0.001$ & 0.446 & $<0.001$ \\
\hline 3 - Disorganized thinking & 0.725 & $<0.001$ & 0.714 & $<0.001$ \\
\hline 4 - Altered level of consciousness & 0.662 & $<0.001$ & - & - \\
\hline Diagnosis of Delirium & 0.853 & $<0.001$ & 0.702 & $<0.001$ \\
\hline
\end{tabular}


same was true in other studies involving different assessment instruments such as Nursing Delirium Symptom Checklist (NuDESC) and Confusion Assessment Method for the Intensive Care Unit (CAM-ICU) done in postoperative patient populations which had an even lower sensitivity. ${ }^{30}$ The contrast was the opposite in the original study of 3D-CAM in which the investigators described a lower specificity instead of sensitivity due to the superimposed effect of delirium on demented elderly patents in medical wards. ${ }^{7,31}$ DSM-5, being the reference standard, has demonstrated a stronger ability to detect subtle changes in mental status compared to less rigorous methods and hence, still the standard of reference. ${ }^{32}$

In this study, the sensitivity for detecting delirium with 3D-CAM was substantially lower at $60 \%$ compared to using M3D-CAM in twothirds of the study population who were able to communicate in both English and Malay. Other than postoperative factors mentioned above, the difference in test performance could reflect lower proficiency in English compared to Malay because in this region, English is commonly the second or third language after Malay and their mothertongue. ${ }^{33}$ The importance of delirium assessment tools that are familiar to the language and culture of patients is therefore, highlighted by this finding.

The M3D-CAM demonstrated excellent interrater reliability of $85 \%$. The clear explanations on how to code each feature from 3D-CAM had been well translated into M3D-CAM to reduce the amount of judgment required by assessors. $^{7}$ The reproducibility across assessors was consistent with high inter-rater agreement among other translated versions of 3D-CAM.,29 Like its original version, the first 10 items in the M3D-CAM were based on patient's direct response while the subsequent 10 were rated based on observation on patient's behaviour. All features 1-4 would be coded as present or absent. To cumulatively diagnose delirium, features 1 and 2 with either one of features 3 or 4 should be present. The agreement for reliability was statistically significant with three features 1,3 \& 4 demonstrating substantial agreement in their $\kappa$-values. Feature 2 was notably the only one with moderate agreement at 0.529 in M3D-CAM and 0.446 in 3D-CAM respectively. ${ }^{3}$

Feature 2 represented inattention, a core characteristic of delirium which was considered positive when patients' answers were incorrect, "don't know" or they had none or nonsensical response towards the items as its indicators. ${ }^{34}$ In 3D-CAM the first 4 items to test for inattention were cognitive tasks with interviewer's instructions to recall numbers or words for weekdays and months backwards followed by 2 items based on interviewer's observation. ${ }^{7}$ The $\kappa$-values in 3D-CAM and M3D-CAM were both moderate indicating no difference between the versions of the items in these two languages. In Malay, the words for days and months are name-specific akin to English, hence no cultural adaptation was required unlike the Chinese language in 3D-CAM-CN. ${ }^{29}$ Another probable explanation was because items 4-7 would have required a higher level of cognitive function to indicate Feature 2 or inattention. ${ }^{34} \mathrm{~A}$ better understanding of instructions, recall of numbers or names and subsequent execution of speech would be needed for these cognitive tasks and because it depended on cognitive function, other factors such as education level would have been linked to a poorer performance and lower agreement. ${ }^{35,36}$ Therefore, to examine the feature of inattention, some studies have suggested to utilize not only a single assessment method but to take it a step further with additional assessment instruments. ${ }^{29,37}$

The strength of this study included having a majority of the study population with bilingual ability. ${ }^{38}$ This allowed parallel testing of M3D$\mathrm{CAM}$ alongside with the original English version by the assessors. A substantial $\kappa$-value of 0.71 was demonstrated, which would otherwise be impossible in previous study populations who could only communicate in a single language. ${ }^{29}$ Conducted in a rigorous design in which the screening instruments and psychiatric examination were administered with results blinded to each assessor, this translated version underwent a highquality evaluation culminating in an assessment with good diagnostics characteristics and agreement for use in a Malay speaking community.

This study has limitations. Assessments were done during daytime and only once per day by each assessor. As with the criteria for a fluctuating course in delirium, some patients' diagnosis could have been missed. Secondly, the present study was performed in a single centre by an experienced trained research team and only enrolled patients after surgery. This may limit the generalizability of our results. We did not examine confounding variables such as pain scores or the effects of prescribed analgesics with sedative effects on the first postoperative day. Furthermore, no stratification of participants' baseline cognitive function was investigated for the analysis of M3D-CAM's psychometric properties that could be affected by pre-existing cognitive impairment. 
In conclusion, despite being a common neuropsychiatric disorder with potentially serious sequelae, the diagnosis of POD is still regrettably not often actively sought in the geriatric population. We report that M3D-CAM is a reliable, sensitive, and specific screening tool for clinicians of non-psychiatric background. Coupled with the ease and brevity of its use, M3D-CAM can be easily incorporated into the daily clinical practice for prompt detection of POD in patients who can speak only Malay.

\section{DISCLOSURE}

Financial support: None

\section{Conflicts of interest: None}

\section{REFERENCES}

1. Moskowitz EE, Overbey DM, Jones TS, et al. Postoperative delirium is associated with increased 5-year mortality. Am J Surg 2017;214(6):1036-8.

2. Moyce Z, Rodseth RN, Biccard BM. The efficacy of peri-operative interventions to decrease postoperative delirium in non-cardiac surgery: a systematic review and meta-analysis. Anaesthesia 2014;69(3):259-69.

3. American Psychiatric Association. Diagnostic and statistical manual of mental disorders. 5th ed. Arlington, VA2013.

4. Inouye SK, Westendorp RG, Saczynski JS. Delirium in elderly people. Lancet 2014;383(9920):911-22.

5. Deiner S, Silverstein JH. Postoperative delirium and cognitive dysfunction. Br J Anaesth 2009;103 Suppl 1(Suppl 1):i41-6.

6. Evered L, Silbert B, Knopman DS, et al. Recommendations for the nomenclature of cognitive change associated with anaesthesia and surgery-2018. Anesth Analg 2018;127(5):1189-95.

7. Marcantonio ER, Ngo LH, O'Connor M, et al. 3D-CAM: derivation and validation of a 3-minute diagnostic interview for CAM-defined delirium: a cross-sectional diagnostic test study. Ann Intern Med 2014;161(8):554-61.

8. The American Geriatrics Society Expert Panel on Postoperative Delirium in Older Adults. American Geriatrics Society abstracted clinical practice guideline for postoperative delirium in older adults. J Am Geriatr Soc 2015;63(1):142-50.

9. Collins N, Blanchard MR, Tookman A, Sampson EL. Detection of delirium in the acute hospital. Age Ageing 2010;39(1):131-5.

10. Shi Q, Warren L, Saposnik G, Macdermid JC. Confusion assessment method: a systematic review and meta-analysis of diagnostic accuracy. Neuropsychiatr Dis Treat 2013;9:1359-70.

11. De J, Wand AP. Delirium screening: A systematic review of delirium screening tools in hospitalized patients. Gerontologist 2015;55(6):1079-99.

12. Wong CL, Holroyd-Leduc J, Simel DL, Straus SE. Does this patient have delirium?: value of bedside instruments. JAMA 2010;304(7):779-86.

13. Beaton DE, Bombardier C, Guillemin F, Ferraz MB. Guidelines for the process of cross-cultural adaptation of self-report measures. Spine (Phila Pa 1976). 2000;25(24):3186-91.

14. Terwee CB, Bot SD, de Boer MR, et al. Quality criteria were proposed for measurement properties of health status questionnaires. J Clin Epidemiol 2007;60(1):34-42.

15. Cheong JL, Shariffuddin II, Danaee M, Khor HM, Teang SC, Loh PS. Understanding risk factors for postoperative delirium after elective surgery in a university-based tertiary hospital. Neurol Asia 2021;26(1):117-27.

16. Arifin WN. Sample size calculator 2021 [February 14, 2021]. Available from: https://wnarifin.github.io/ ssc/sssnsp.html.

17. Buderer NM. Statistical methodology: I. Incorporating the prevalence of disease into the sample size calculation for sensitivity and specificity. Acad Emerg Med 1996;3(9):895-900.

18. Landis JR, Koch GG. The Measurement of observer agreement for categorical data. Biometrics 1977;33(1):159-74.

19. Iamaroon $A$, Wongviriyawong $T$, Sura-Arunsumrit $P$, Wiwatnodom N, Rewuri N, Chaiwat O. Incidence of and risk factors for postoperative delirium in older adult patients undergoing noncardiac surgery: a prospective study. BMC Geriatr 2020;20(1):40.

20. Strøm C, Rasmussen LS. Challenges in anaesthesia for elderly. Singapore Dent J 2014;35c:23-9.

21. Siddiqi N, House AO, Holmes JD. Occurrence and outcome of delirium in medical in-patients: a systematic literature review. Age Ageing 2006;35(4):350-64.

22. Kiely DK, Jones RN, Bergmann MA, Marcantonio ER. Association between psychomotor activity delirium subtypes and mortality among newly admitted post-acute facility patients. $J$ Gerontol A Biol Sci Med Sci 2007;62(2):174-9.

23. Yang FM, Marcantonio ER, Inouye SK, et al. Phenomenological subtypes of delirium in older persons: patterns, prevalence, and prognosis. Psychosomatics 2009;50(3):248-54.

24. Witlox J, Eurelings LS, de Jonghe JF, Kalisvaart KJ, Eikelenboom P, van Gool WA. Delirium in elderly patients and the risk of postdischarge mortality, institutionalization, and dementia: a meta-analysis. JAMA 2010;304(4):443-51.

25. Cole MG, Primeau FJ. Prognosis of delirium in elderly hospital patients. CMAJ 1993;149(1):41-6.

26. Inouye SK, van Dyck CH, Alessi CA, Balkin S, Siegal AP, Horwitz RI. Clarifying confusion: the confusion assessment method. A new method for detection of delirium. Ann Intern Med 1990;113(12):941-8.

27. Neufeld KJ, Yue J, Robinson TN, Inouye SK, Needham DM. Antipsychotic medication for prevention and treatment of delirium in hospitalized adults: A systematic review and meta-analysis. J Am Geriatr Soc 2016;64(4):705-14.

28. Greysen SR. Delirium and the "know-do" gap in acute care for elders. JAMA Intern Med 2015;175(4):521-2.

29. Mu DL, Ding PP, Zhou SZ, et al. Cross-cultural 
adaptation and validation of the 3D-CAM Chinese version in surgical ICU patients. BMC Psychiatry 2020;20(1):133.

30. Neufeld KJ, Leoutsakos JS, Sieber FE, et al. Evaluation of two delirium screening tools for detecting post-operative delirium in the elderly. $\mathrm{Br}$ J Anaesth 2013;111(4):612-8.

31. Fick DM, Agostini JV, Inouye SK. Delirium superimposed on dementia: a systematic review. $J$ Am Geriatr Soc 2002;50(10):1723-32.

32. Rudolph JL, Marcantonio ER. Review articles: postoperative delirium: acute change with long-term implications. Anesth Analg 2011;112(5):1202-11.

33. Awal NM, Jaafar MF, Mis MA, Lateh H. Maintenance of mother tongue: Patterns of language choice at the Malaysian-Thai border. Procedia - Social and Behavioral Sciences 2014;118:282-7.

34. Huang LW, Inouye SK, Jones RN, et al. Identifying indicators of important diagnostic features of delirium. J Am Geriatr Soc 2012;60(6):1044-50.

35. Pengpid S, Peltzer K, Susilowati IH. Cognitive functioning and associated factors in older adults: Results from the Indonesian family life survey-5 (IFLS-5) in 2014-2015. Curr Gerontol Geriatr Res 2019;2019:4527647.

36. Din NC, Shahar S, Zulkifli BH, Razali R, Chyn AV, Omar A. Validation and optimal cut-off scores of the bahasa Malaysia version of the montreal cognitive assessment (MoCA-BM) for mild cognitive impairment among community dwelling older adults in Malaysia. Sains Malaysiana 2016;45(9):1337-43.

37. Katzman R, Zhang MY, Ouang Ya Q, et al. A Chinese version of the Mini-Mental State Examination; impact of illiteracy in a Shanghai dementia survey. J Clin Epidemiol 1988;41(10):971-8.

38. Chan SH, Abdullah AN. Bilingualism in Malaysia: language education policy and local needs. Pertanika Journal of Social Sciences and Humanities 2015;23(3):55-70. 